\title{
A BOUNDED MAP ASSOCIATED TO A ONE-PARAMETER GROUP OF *-AUTOMORPHISMS OF A VON NEUMANN ALGEBRA
}

\author{
by A. B. THAHEEM
}

(Received 4 November, 1982)

1. Introduction. Let $M$ be a von Neumann algebra and $\left\{\alpha_{t}: t \in \mathbf{R}\right\}$ a strongly continuous one-parameter group of *-automorphisms of $M$. Recently, considerable interest has been shown in the associated mappings $\left\{\alpha_{t}+\alpha_{-t}: t \in \mathbf{R}\right\}$ (see for instance [2], [3] and [4]). The paper of Thaheem, Van Daele and Vanheeswijk [4] exclusively deals with these mappings. The main result of [4] is formulated as follows. Let $\left\{\alpha_{t}: t \in \mathbf{R}\right\}$ and $\left\{\beta_{t}: t \in \mathbf{R}\right\}$ be strongly continuous one-parameter groups of ${ }^{*}$-automorphisms on a von Neumann algebra $\boldsymbol{M}$ satisfying the operator equation

$$
\alpha_{t}+\alpha_{-t}=\beta_{t}+\beta_{-t} \quad(t \in \mathbf{R}) .
$$

If $M$ is a factor then either $\alpha_{t}=\beta_{t}$ or $\alpha_{t}=\beta_{-t}$, for all $t \in \mathbf{R}$. In general, there exists a central projection $p$ in $M$ such that $\alpha_{t}(x)=\beta_{t}(x)$ for all $x \in M p$ and

$$
\alpha_{t}(x)=\beta_{-t}(x) \text { for all } x \in M(1-p) .
$$

In fact, in a recent paper of Haagerup and Skau [2], the above operator equation (in the case of modular automorphisms) has played a crucial role in the geometric interpretation of the Tomita-Takesaki theory. The purpose of this paper is to develop a suitable functional calculus associated with an automorphism group $\left\{\alpha_{t}: t \in \mathbf{R}\right\}$ which reconstructs the mappings $\left\{\alpha_{t}+\alpha_{-t}: t \in \mathbf{R}\right\}$. To any strongly continuous one-parameter group of *automorphisms $\left\{\alpha_{t}: t \in \mathbf{R}\right\}$ of a von Neumann algebra $M$, we associate a linear map $\psi$ of $M$ by the formula

$$
\psi(x)=\int_{-\infty}^{+\infty} \frac{2}{e^{\pi t}+e^{-\pi t}} \alpha_{t}(x) d t, \quad(x \in M) .
$$

We show that $\psi$ determines the automorphism group almost completely in the sense that if $\left\{\alpha_{t}: t \in \mathbf{R}\right\}$ and $\left\{\beta_{t}: t \in \mathbf{R}\right\}$ are strongly continuous one-parameter groups of $*_{\text {- }}$ automorphisms of $M$ with $\psi_{\alpha}$ and $\psi_{\beta}$ as the corresponding maps, then $\psi_{\alpha}=\psi_{\beta}$ implies that $\alpha_{t}+\alpha_{-t}=\beta_{t}+\beta_{-t}$ for all $t \in \mathbf{R}$. In other words $\psi_{\alpha}=\psi_{\beta}$ implies that $\alpha_{t}=\beta_{t}$ on $M p$ and $\alpha_{t}=\beta_{-t}$ on $M(1-p)$ for a central projection $p$ in $M([4])$.

We believe that in general the mapping $\psi$ may serve as a tool to study one-parameter groups of *automorphisms similar to the infinitesimal generator. It has less algebraic properties than the infinitesimal generator, but it has the advantage of being a bounded map. $\psi$ is injective and completely positive. Some simple properties of $\alpha$ are reflected in simple properties of $\psi$. For example, an element $x \in M$ is a fixed point of $\alpha$; that is $\alpha_{\mathrm{t}}(x)=x$ for all $t \in \mathbf{R}$ if and only if $\psi(x)=x$.

Glasgow Math. J. 25 (1984) 135-140. 
Our original motivation in introducing the mapping $\psi$ comes from the paper of Van Daele on the new approach of the Tomita-Takesaki theory, where in the case of modular automorphism groups all this was related to the simple formula

$$
\frac{2 \Delta^{1 / 2}}{\Delta+1}=\int_{-\infty}^{+\infty} \frac{2}{e^{\pi t}+e^{-\pi t}} \Delta^{i t} d t
$$

where $\Delta$ is the modular operator ([5]).

We are grateful to the referee for drawing our attention to the paper of Ciorănescu and Zsidó [1] in which the authors introduce the "analytic generator" of a continuous group of operators on a Banach space. Our map $\psi$ is similar to their "analytic generator". Also our main result can alternatively be proved by using their inversion formula and our Lemma 2.6.

2. Results. We assume that the von Neumann algebra $M$ is acting on a Hilbert space $H$ and $B(H)$ is the algebra of bounded linear operators on $H$.

Definition 2.1. Let $M$ be a von Neumann algebra and $\left\{\alpha_{t}: t \in \mathbf{R}\right\}$ be a one-parameter group of *automorphisms of $M$. Define a map $\psi$ on $M$ by the formula

$$
\psi(x)=\int_{-\infty}^{+\infty} \frac{2}{e^{\pi t}+e^{-\pi t}} \alpha_{t}(x) d t, \quad(x \in M)
$$

Clearly, the integral is well-defined in the strong operator topology and gives a bounded linear operator on $H$. If we want to specify that $\psi$ is associated to $\left\{\alpha_{\mathbf{t}}: t \in \mathbf{R}\right\}$, we shall write it as $\psi_{\alpha}$.

Some properties of $\psi$ are immediate from the definition. Since

$$
\int_{-\infty}^{+\infty} \frac{2}{e^{\pi t}+e^{-\pi t}} d t=1
$$

$\|\psi\| \leqslant 1$ and $\psi(1)=1$. So $\|\psi\|=1$.

Considering any element $y$ in the commutant $M^{\prime}$ of $M$, it is easy to see that $\psi(x)$ commutes with $y$ and hence $\psi(x) \in M$. Therefore, $\psi$ is a mapping of $M$ into $M$.

Proposttion 2.2. $\psi$ is completely positive.

Proof. We first observe that *-automorphisms $\alpha_{t}$ carry positive elements into positive elements. Let $a_{i j} \in M$ and $\sum_{i, j} a_{i j} \otimes e_{i j} \geqslant 0$, where $e_{i j}$ denotes the matrix with elements equal to 0 , except the $i, i$-element which is equal to 1 . Then

$$
\begin{aligned}
\left(\psi \otimes 1_{n}\right)\left(\sum_{i, j}\left(a_{i j} \otimes e_{i j}\right)\right) & =\sum_{i, j} \psi\left(a_{i j}\right) \otimes e_{i j} \\
& =\sum_{i, j} \int_{-\infty}^{+\infty} \frac{2}{e^{\pi t}+e^{-\pi t}}\left(\alpha_{i} \otimes 1_{n}\right)\left(a_{i j} \otimes e_{i j}\right) d t \geqslant 0
\end{aligned}
$$

for all integers $n \geqslant 1$.' Thus $\psi$ is completely positive. 
To prove our main result and a few other properties of $\psi$, we need some lemmas.

LEMMA 2.3. For any $r>0$ and $s \in \mathbf{R}$,

$$
\int_{-\infty}^{+\infty} \frac{r^{i t}}{e^{\pi t}+e^{-\pi t}} e^{-i s t} d t=\frac{e^{s / 2} r^{1 / 2}}{e^{s}+r}
$$

Proof. Replacing $e^{-\mathrm{s}}$ by $e^{-\mathrm{s}} r$ we obtain the general case from $r=1$. So we will assume $r=1$. Consider the complex-valued function

$$
f(z)=\frac{e^{-i s z}}{e^{\pi z}+e^{-\pi z}}
$$

Then

$$
\lim _{z \rightarrow i / 2}[(z-i / 2) f(z)]=\frac{e^{s / 2}}{2 \pi i} .
$$

Integrating $f(z)$ over a rectangle joining the points $\pm R, \pm R+i$ (which contains a pole of $f(z)$ at $z=i / 2),|f(z)| \rightarrow 0$ as $R \rightarrow \infty$ and $\operatorname{Im} z$ remains bounded; we get

$$
\int_{-\infty}^{+\infty} f(t) d t-\int_{-\infty}^{+\infty} f(t+i) d t=e^{s}
$$

Evaluating these integrals, we obtain the desired result.

Lemma 2.4. Let $M$ be a von Neumann algebra and $\left\{\alpha_{t}: t \in \mathbf{R}\right\}$ be a group of *automorphisms of $M$. For any $f \in L_{1}(\mathbf{R})$, define a mapping $\phi(f)$ from $M$ into $M$ by

$$
\phi(f) x=\int_{-\infty}^{+\infty} f(t) \alpha_{t}(x) d t \quad(x \in M)
$$

Then $\phi$ is linear and for all $f$ and $g$ in $L_{1}(\mathbf{R}), \phi(f) \phi(g)=\phi(f * g)$. Furthermore $\phi(f)$ is bounded and continuous for the $\sigma\left(M, M_{*}\right)$ topology on $M$.

Proof. For a proof we refer to [6].

DeFinITION 2.5. For any $r>0$ define an operator $\psi^{(r)}$ on $M$ by

$$
\psi^{(r)}(x)=\int_{-\infty}^{+\infty} \frac{2 r^{i t}}{e^{\pi t}+e^{-\pi t}} \alpha_{t}(x) d t, \quad(x \in M) .
$$

As before $\psi^{(r)}$ is a bounded operator which maps $M$ into $M$.

LeMma 2.6. For any $r>0$, we have

$$
\left(1+\left(\frac{r^{1 / 2}-r^{-1 / 2}}{2}\right)^{2} \psi^{2}\right)\left(\psi^{(r)}+\psi^{\left(r^{-1}\right)}\right)=\left(r^{1 / 2}+r^{-1 / 2}\right) \psi
$$

Proof. Put

$$
f_{r}(t)=\frac{2 r^{i t}}{e^{\pi t}+e^{-\pi t}}, \quad f_{r^{-1}}(t)=\frac{2 r^{-i t}}{e^{\pi t}+e^{-\pi t}}, \quad f(t)=\frac{2}{e^{\pi t}+e^{-\pi t}} .
$$


By Lemma 2.4. it is enough to prove that

$$
\left(1+\left(\frac{r^{1 / 2}-r^{-1 / 2}}{2}\right)^{2} \hat{f}^{2}\right)\left(\hat{f}_{r}+\hat{f}_{r^{-1}}\right)=\left(r^{1 / 2}+r^{-1 / 2}\right) \hat{f}
$$

where as usual $\hat{f}$ denotes the Fourier transform of $f$. From Lemma 2.3. we have that the left hand side of equation (1) is equal to

$$
\begin{aligned}
\left(1+\left(\frac{r^{1 / 2}-r^{-1 / 2}}{2}\right)^{2} \hat{f}^{2}\right)\left(2 e ^ { s / 2 } \left(\frac{r^{1 / 2}}{e^{s}+r}\right.\right. & \left.\left.+\frac{r^{-1 / 2}}{e^{s}+r^{-1}}\right)\right) \\
& =2\left(1+\left(\frac{r^{1 / 2}-r^{-1 / 2}}{2}\right)^{2} \hat{f}^{2}\right) \frac{\left(e^{s / 2}+e^{-s / 2}\right)\left(r^{1 / 2}+r^{-1 / 2}\right)}{\left(e^{s / 2}+e^{-s / 2}\right)^{2}+\left(r^{1 / 2}-r^{-1 / 2}\right)^{2}} \\
& =2\left(1+\left(\frac{r^{1 / 2}-r^{-1 / 2}}{2}\right)^{2} \hat{f}^{2}\right) \frac{2 \hat{f}^{-1}\left(r^{1 / 2}+r^{-1 / 2}\right)}{4 \hat{f}^{-2}+\left(r^{1 / 2}-r^{-1 / 2}\right)^{2}} \\
& =\left(1+\left(\frac{r^{1 / 2}-r^{-1 / 2}}{2}\right)^{2} \hat{f}^{2}\right) \frac{\left(r^{1 / 2}+r^{-1 / 2}\right) \hat{f}}{\left(1+\left(\frac{r^{1 / 2}-r^{-1 / 2}}{2}\right)^{2} \hat{f}^{2}\right)} \\
& =\left(r^{1 / 2}+r^{-1 / 2}\right) \hat{f} .
\end{aligned}
$$

This proves the lemma.

LEMMA 2.7. For any $r>0$, the operator

$$
\left(1+\left(\frac{r^{1 / 2}-r^{-1 / 2}}{2}\right)^{2} \psi^{2}\right)
$$

is injective.

Proof. Let $x \in M$ and suppose

$$
\left(1+\left(\frac{r^{1 / 2}-r^{-1 / 2}}{2}\right)^{2} \psi^{2}\right)(x)=0
$$

From Lemma 2.6. and the fact that all the operators $\psi^{(r)}$ and $\psi$ commute, we conclude that $\psi(x)=0$ and $\psi^{2}(x)=0$. It follows from equation (2) that $x=0$.

Proposmion 2.8. $\psi$ is injective.

Proof. Let $x \in M$ and suppose $\psi(x)=0$. Then $\psi^{2}(x)=0$ and by Lemma 2.6. we have

$$
\left(\psi^{(r)}+\psi^{(r-1)}\right)(x)=0
$$

So

$$
\int_{-\infty}^{+\infty} \frac{r^{i t}}{e^{\pi t}+e^{-\pi t}} \alpha_{t}(x) d t+\int_{-\infty}^{+\infty} \frac{r^{-i t}}{e^{\pi t}+e^{-\pi t}} \alpha_{t}(x) d t=0
$$

or

$$
\int_{-\infty}^{+\infty} \frac{r^{i t}}{e^{\pi t}+e^{-\pi t}}\left(\alpha_{t}(x)+\alpha_{-t}(x)\right) d t=0
$$


Put $r=e^{-s}$ and

$$
g(t)=\frac{1}{e^{\pi t}+e^{-\pi t}}\left\langle\left(\alpha_{t}(x)+\alpha_{-t}(x)\right) \xi, \eta\right\rangle
$$

for a given pair $\xi, \eta$ in $H$. Then $\hat{g}(s)=0$ for all $s$. By continuity $g=0$ and since this holds for all $\xi$ and $\eta$, we have $\alpha_{t}(x)+\alpha_{-t}(x)=0$ for all $t \in \mathbf{R}$ and hence $x=0$.

We are now ready to prove our main result.

THEOREM 2.9. Let $\left\{\alpha_{\mathrm{t}}: t \in \mathbf{R}\right\}$ and $\left\{\beta_{\mathrm{t}}: t \in \mathbf{R}\right\}$ be two one-parameter groups of *automorphisms of a von Neumann algebra $M$. If $\psi_{\alpha}(x)=\psi_{\beta}(x)$, then for every $x$ in $M$

$$
\alpha_{t}(x)+\alpha_{-t}(x)=\beta_{t}(x)+\beta_{-t}(x) \text { for all } t \in \mathbf{R} \text {. }
$$

Proof. Let $\psi_{\alpha}(x)=\psi_{\beta}(x)=\psi(x)$. Then for any $r>0$, Lemma 2.6. and the fact that $\psi^{(r)}$ and $\psi$ commute together imply that

$$
\left(1+\left(\frac{r^{1 / 2}-r^{-1 / 2}}{2}\right)^{2} \psi^{2}\right)\left(\psi_{\alpha}^{(r)}+\psi_{\alpha}^{(r-1)}\right)(x)=\left(1+\left(\frac{r^{1 / 2}-r^{-1 / 2}}{2}\right)^{2} \psi^{2}\right)\left(\psi_{\beta}^{(r)}+\psi_{\beta}^{\left(r^{-1}\right)}\right)(x)
$$

By Lemma 2.7.,

$$
\left(1+\left(\frac{r^{1 / 2}-r^{-1 / 2}}{2}\right)^{2} \psi^{2}\right)
$$

is injective and hence

$$
\left(\psi_{\alpha}^{(r)}+\psi_{\alpha}^{\left(r^{-1}\right)}\right)(x)=\left(\psi_{\beta}^{(r)}+\psi_{\beta}^{\left(r^{-1}\right)}\right)(x) .
$$

By the argument of Proposition 2.8. it follows that $\alpha_{t}(x)+\alpha_{-t}(x)=\beta_{t}(x)+\beta_{-t}(x)$ for all $t \in \mathbf{R}$.

We conclude the paper with the following fixed point theorem.

Theorem 2.10. Let $x \in M$. Then $\psi(x)=x$ if and only if $\alpha_{t}(x)=x$ for all $t \in \mathbf{R}$.

Proof. If $\alpha_{t}(x)=x$ for all $t \in \mathbf{R}$, then $\psi(x)=x$ because

$$
\int_{-\infty}^{+\infty} \frac{2}{e^{\pi t}+e^{-\pi t}} d t=1
$$

Conversely if $\psi(x)=x$, taking $\beta_{t}$ to be the identity in Theorem 2.9 , we obtain

$$
\alpha_{t}(x)+\alpha_{-t}(x)=2 x \quad(t \in \mathbf{R}) .
$$

This implies that

$$
\left(\alpha_{t}-1\right)\left(\alpha_{t}-1\right)(x)=0 .
$$

Put $\left(\alpha_{t}-1\right)(x)=y$. Then $\alpha_{t}(y)=y$ or $\alpha_{n t}(y)=y$ for every integer $n$. But $\alpha_{t}(x)=x+y$, so 
that $\alpha_{n t}(x)=x+n y$. This means that

$$
n\|y\| \leqslant\left\|\alpha_{n t}(x)\right\|+\|x\| \leqslant 2\|x\|
$$

for all integers $n$. So $y=0$ or $\alpha_{t}(x)=x$ for all $t \in \mathbf{R}$.

Acknowledgments. We wish to express our sincere thanks to Professor A. Van Daele for his help during this research. We are also grateful to the referee for useful suggestions which improved the paper.

\section{REFERENCES}

1. I. Ciorănescu and L. Zsidó, Analytic generators for one-parameter groups, Tôhoku Math. J. 28 (1976), 327-362.

2. U. Haagerup and C. F. Skau, Geometric aspects of the Tomita-Takesaki theory II, Math. Scand. 48 (1981), 241-252.

3. A. B. Thaheem, On a decomposition of a von Neumann algebra, Rend. Sem. Mat. Univ. Padova 65 (1981), 1-7.

4. A. B. Thaheem, A. Van Daele and L. Vanheeswijck, A result on two one-parameter groups of automorphisms. Math. Scand. 51 (1982), 261-274.

5. A. Van Daele, A new approach to the Tomita-Takesaki theory of generalized Hilbert algebras, J. Functional Analysis 15 (1974), 378-393.

6. A. Van Daele, Arveson's theory of spectral subspaces, Nieuw Arch. Wisk. 27 (1979), 215-237.

Permanent address:

249, G-8/1,

IsLAMABAD, PAKISTAN.

Present address:

Faculty of SCIENCE,

DeparTMENT OF MATHEMATICS,

GARYOUNIS UNIVERSITY,

P.O. BOX 9480,

BenghazI, Libya. 